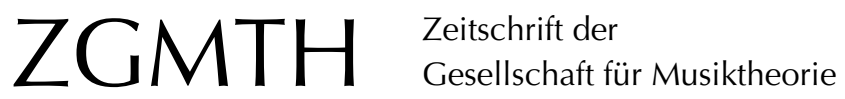

Menke, Johannes (2010): Brauchen wir einen Kanon in der Musiktheorie? ZGMTH 7/1, 61-70. https://doi.org/10.31751/507

(C) 2010 Johannes Menke

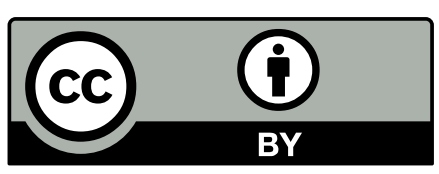

Dieser Text erscheint im Open Access und ist lizenziert unter einer Creative Commons Namensnennung 4.0 International Lizenz.

This is an open access article licensed under a

Creative Commons Attribution 4.0 International License.

veröffentlicht / first published: 01/02/2010

zuletzt geändert / last updated: 13/10/2011 


\title{
Brauchen wir einen Kanon in der Musiktheorie?
}

\author{
Johannes Menke
}

Manfred Fuhrmanns Konzeption von Bildung als Teilhabe an Kultur aufgreifend, wird diskutiert, welche Rolle ein Kanon in der Musiktheorie spielen könnte bzw. müsste. Die Funktion des Kanons wäre es, Teilhabe an der Kultur der Musik oder im Falle tonaler Musik an der Kultur der Tonalität zu ermöglichen. Hierbei ist ein Kanon der Kompetenzen und der Werke zu unterscheiden. Der Autor plädiert dafür, die Konzentration auf komplexe Meisterwerke weniger Komponisten zu hinterfragen und stattdessen an historische Traditionen kompositorischen und improvisatorischen Handwerks anzuknüpfen.

\section{I.}

Das Thema `Kanon` hat in den letzten Jahren Aufmerksamkeit erfahren. Bücher, in denen steht, 'was man wissen muss $\iota^{2}$, standen auf den Bestseller-Listen, bedeutende Literaturkritiker wie Marcel Reich-Ranicki ${ }^{3}$ veröffentlichten einen Kanon der Literatur, und auch die Zeitschrift Musiktheorie: Zeitschrift für Musikwissenschaft hatte in einer Ausgabe aus dem Jahr 2006 das Thema 'Kanon` als Schwerpunkt. ${ }^{4}$ Die Diskussion über den Kanon indiziert nicht seine Überholtheit, wie manche meinen, sondern einen vorhandenen Klärungsbedarf.

Als auf dem V. Kongress der GMTH in Hamburg (2005) bei einer Podiumsdiskussion beiläufig konstatiert wurde, dass wir im Musiktheorieunterricht selbstverständlich keinen Kanon hätten, widersprach niemand. Mich machte diese Bemerkung so nachdenklich, dass ich nicht sofort reagieren mochte und mit den folgenden Ausführungen sozusagen verzögert antworte. Die folgenden Ausführungen sind daher nicht als wissenschaftliche Abhandlung zu verstehen, sondern als verspäteter Diskussionsbeitrag.

1 Diesen Text hielt ich als Vortrag auf dem VI. Kongress der GMTH 2006 in Weimar. Hier handelt es sich um eine leicht überarbeitete Fassung. Manche der vorgebrachten Auffassungen mögen mittlerweile und mancherorts schon verbreiteter sein als vor drei Jahren, das Thema scheint mir aber nach wie vor aktuell zu sein.

2 Schwanitz 1999.

3 Reich-Ranicki 2002.

4 Musiktheorie. Zeitschrift für Musikwissenschaft 1/2006. 
II.

Allen gegenteiligen Bemühungen zum Trotz gilt die Musiktheorie mancherorts immer noch als trockene Disziplin. Wir wollen nicht vertiefen, wie es, vor allem unter Kollegen in den Instrumentalfächern, zu einer solchen Einschätzung kommen mag, das Postulat eines Kanons scheint jedenfalls auf den ersten Blick der Attraktivität unseres Faches nicht gerade zuträglich zu sein, denn es klingt nach Verschulung und Einschränkung. Nun muss zunächst ganz nüchtern festgehalten werden, dass es ja an allen Hochschulen einen Kanon geben muss, dergestalt dass bestimmte Dinge in der Prüfungsordnung verankert sind, bzw. dass jeder Lehrer entscheiden muss, was im Unterricht behandelt werden soll. Eine gewisse Kanonbildung ist also aus rein pragmatischen Gründen überhaupt nicht zu vermeiden, und in der Tat gibt es verbreitete Vorlieben: so die Musik von Bach im Allgemeinen (Johann Sebastian, versteht sich), der Bach-Choral, die Bachsche Fuge, das Bachsche Choralvorspiel, der Bachsche Suitensatz etc., des weiteren die BeethovenSonaten, Schubert-Lieder usw. Darüber hinaus hat jeder (zurecht) seinen Privat-Kanon und fürchtet womöglich, ein allgemein-verbindlicher Kanon könnte seine persönliche Freiheit beeinträchtigen.

Ich frage mich aber: Müssen wir die Frage danach, was wir unterrichten, nicht immer wieder neu stellen? Haben wir nicht die Pflicht unseren Schülern gegenüber, genau auszuwählen, was für sie wichtig ist? Muss es nicht etwas zwischen dem Trott der Routine und der bequemen und vermeintlich politisch korrekten Haltung des sanything goes geben?

Was aber ist ein Kanon und wozu braucht man ihn? Es sei gestattet, einige prinzipielle Gedanken vorauszuschicken, bevor wir wieder zur Musiktheorie kommen, denn es soll in diesem Text nicht darum gehen, einen ausgearbeiteten Kanon zu präsentieren, sondern über seine Möglichkeiten und Bedingungen nachzudenken.

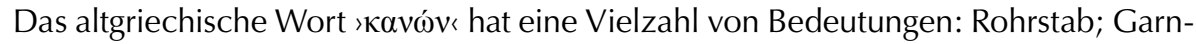
spule (!); Richtschnur; Vorschrift, Regel, Norm, Vorbild. ${ }^{5}$ Die Etymologie macht den normativen und exemplarischen Charakter des Kanons deutlich, erklärt aber noch nicht seine Funktion.

Hinter der Frage nach dem Kanon steht die Frage nach der Bildung. Der Altphilologe Manfred Fuhrmann hat wenige Jahre vor seinem Tod ein eindrucksvolles Buch mit dem Titel Bildung - Europas kulturelle Identität geschrieben. ${ }^{6}$ Für Fuhrmann ist klar, dass die Konzeption von Bildung nicht von ihrer Geschichte, von ihren Inhalten und dem übergeordneten Kulturbegriff getrennt werden kann. Es versteht sich von selbst, dass der Frage nach Bildung die Frage nach dem Kanon auf den Fuß folgt:

Die beiden Aspekte, Bildungskanon und Bildungsidee gehören zusammen wie Topf und Deckel; sie dürfen indes nicht, wie es bisweilen geschieht, einander gleichgesetzt oder miteinander verwechselt werden. ${ }^{7}$

5 Gemoll 1988, 407.

6 Fuhrmann 2002

7 Ebd., 35. 
Kanon, Bildung und Kultur sind drei Kategorien, die in einer hierarchischen Beziehung zueinander stehen: Zuoberst steht die Kultur, Bildung ist die Teilhabe des einzelnen an der Kultur, der Kanon wiederum soll gewissermaßen mit der Funktion eines kollektiven Schlüssels dem einzelnen diese Teilhabe ermöglichen.

Ein Kanon ist eine wertende Auswahl aus einem größeren Ganzen, aus einem Inbegriff vergleichbarer Gegebenheiten, getroffen von einer autoritativen Instanz und stabil über die Zeiten hinweg; das größere Ganze aber ist beim Bildungskanon nicht die Bildung, sondern die Kultur. ${ }^{8}$

»Man müsste«, so Fuhrmann weiter, »eigentlich `Kulturkanon` sagen«. Fuhrmann weist darauf hin, dass die begriffliche Unterscheidung von `Kultur` und `Bildung « eine Eigenart der deutschen Sprache sei. Dies stehe jedoch nicht unbedingt für einen deutschen Sonderweg, solange man die gegenseitige Verwiesenheit der beiden Begriffe im Auge behalte. `Bildung، gehe zurück auf Platons Konzeption der `Methexis`, also der `Teilhabe`. Mithilfe des Kanons erlangt der einzelne `Teilhabe` an der Kultur, je repräsentativer die Auswahl des Kanons, desto gelungener die Teilhabe. Kultur aber bleibt etwas, was den einzelnen immer übersteigen wird:

[...] Kultur ist `Potenz im doppelten Sinn: als gegebener, so oder so beschaffener Horizont, der in allen seinen Bereichen die ständige Produktion von Neuem ermöglicht, und als Summe des Überlieferten, als Inbegriff alles dessen, was in Bibliotheken, Archiven, Magazinen und sonstigen Speichern bewahrt wird, unabhängig davon, ob die jeweils Lebenden noch etwas damit anzufangen wissen oder nicht. ${ }^{9}$

Der doppelten Potentialität der Kultur gegenüber steht die Aktualität des Kanons, letztes Fuhrmann-Zitat:

Der 'Kanon` sucht zwischen der unüberschaubaren Vielfalt der Kultur und den einzelnen, die an ihr teilhaben, zu vermitteln: Er reduziert die Potenzialität auf Aktualität, auf eine für das einzelne Subjekt überschaubare Auswahl. Er erlangt, da er auf anerkannten Normen beruht, über eine längere Zeit hinweg für eine ganze Gruppe oder Schicht von Subjekten Gültigkeit. Er ist keine starre, unveränderliche Größe; im Gegenteil, er kann stets durch Rückgriffe auf bis dahin latente Überlieferung sowie durch Zugang von neu Produziertem modifiziert werden. ${ }^{10}$

Fassen wir noch einmal zusammen: Durch die Aktualität des Kanons erlangt der einzelne Teilhabe an der doppelten Potentialität der Kultur. Der Prozess dieser Teilhabe kann mit dem Begriff `Bildung` beschrieben werden. Der Kanon impliziert Dinge, die heute für mache problematisch geworden sind: Er impliziert Normen, Wertung und Orientierung, daher womöglich die Abwehrhaltung gegen den Kanon. Die Normen setzen voraus, was prinzipiell erstrebenswert ist, die Wertung ist notwendig, um eine Auswahl

8 Ebd., 37.

9 Ebd., $40 \mathrm{f}$.

10 Ebd., 41. 
zu treffen und die Orientierung steht für die Überzeugung, dass es um mehr geht als bloßes Know-how. Wichtig ist aber auch die Modifizierbarkeit des Kanons: Zwar ist der Kanon nur dann sinnvoll, wenn er eine gewisse Gültigkeit beanspruchen kann, er muss aber dennoch durch Rückgriffe und Neuzugänge ständig revidiert werden, um seine Gültigkeit zu wahren.

Letztlich steht und fällt ein Kanon mit dem ihm übergeordneten Kulturbegriff. Ohne Kulturbegriff kein Bildungsbegriff und infolgedessen auch keinen Kanon. Über den Kulturbegriff lässt sich freilich trefflich streiten; so lange wir uns aber einig sind, dass die Auseinandersetzung mit einem Monteverdi-Madrigal, einer Mozart-Sinfonie, einer Wagner-Oper oder einem Klavierstück von Ligeti lohnenswert ist, setzen wir musikalische Kultur voraus. Der Tatsache, dass der überwiegende Teil der Musik, mit der wir uns beschäftigen, historisch ist, sollte nicht mit verlegenen Hinweisen auf die Aktualität der Werke ausgewichen werden: Im Gegenteil, eine offensive Bejahung der Historizität vermag das Alte zu einer Quelle für neue Erfahrungen zu machen. Diese Lektion kann man aus den letzten 30 Jahren Historische Aufführungspraxis lernen: Paradoxerweise gewinnt Vergangenes dann an Aktualität, wenn man es nicht teleologisch deutet, sondern sich darum bemüht zu verstehen, wie es wirklich gewesen ist. Nicht mit dem Hinweis darauf, wie revolutionär ein Werk, oder wie sehr es seiner Zeit voraus gewesen sei, kommt man weiter, sondern dadurch, dass man der Vergangenheit mit einer Neugierde und Wissbegierde entgegentritt, wie sie die Renaissance der Antike entgegenbrachte.

III.

Musiktheorie spielt bei der musikalischen Bildung eine zentrale Rolle. Es ist ein Kernfach der musikalischen Bildung. Die Musiktheorie ermöglicht Teilhabe im doppelten Sinn: Sie vermittelt musikalisch-technische Kompetenzen, lapidar gesagt die Fähigkeit, Musik hören, lesen, schreiben und erfinden zu können. Daneben geht es ihr darum, Musikwerke verschiedener Epochen zu verstehen, also ihre Faktur zu beschreiben und ihren kompositionstechnischen, kulturellen, religiösen, politischen, sozialen, wirtschaftlichen Hintergrund zu berücksichtigen.

In beiden Fällen muss eine Auswahl getroffen werden, es gibt also in der Musiktheorie einen Kanon der zu erwerbenden Kompetenzen und einen Kanon der zu analysierenden Werke. Selbstverständlich steht beides in inhaltlichem Zusammenhang, es sind aber zwei operativ getrennte Bereiche.

Widmen wir uns zunächst dem Kanon der Kompetenzen. Musik hören, lesen, schreiben und erfinden lernen kann man nicht san sichı, sondern nur in Bezug auf einen bestimmten stilistischen Kontext. Ein Schwerpunkt unserer Arbeit an den meisten Instituten ist sicherlich die europäische Kunstmusik der harmonischen Tonalität, grob gesprochen die Zeit von 1600 bis 1900.

Ungeachtet der stilistischen Unterschiede gibt es in diesem Zeitraum tatsächlich eine `Tonalität an sich`, man könnte sagen eine Kultur der Tonalität mitsamt ihrer Differenzierungen und ihrer Geschichte. Worin aber besteht diese Kultur? Ich stütze mich im Folgenden auf einen Grundsatzartikel zur Tonalität von Michael Polth, den dieser 2001 in 
der Zeitschrift Musik \& Ästhetik veröffentlicht hat. ${ }^{11}$ Tonalität, so Polth, setze eine »Harmonie der Umstände « voraus $^{12}$, sie ist gleichsam ein kognitives Medium, das Werk und Hörerschaft gleichermaßen umfasst:

Als ganze gibt es sie [die Tonalität] nur, wenn ein kompetenter Hörer ein tonales Werk hört. ${ }^{13}$

Tonalität beruht, so Polth sinngemäß weiter, nicht auf naturwissenschaftlich fundierten Axiomen, wie Rameau und das 19. Jahrhundert glaubten, sondern auf einem Usus, der sich in herausragenden Werken der sauctores aprobati manifestiert. Konstitutiv für Tonalität sind demnach nicht wenige Urformeln, wie etwa die drei Hauptfunktionen, der Ursatz oder dergleichen, sondern der Usus, also eine Vielzahl von technischen Standards, Vorformatierungen und Regelmäßigkeiten. Der tonale Usus schlägt sich nieder in musikalischen Mustern, Modellen und Topoi, die sich über Jahrhunderte hinweg als tonale Konstanten dingfest machen lassen.

Individuation, so Polth in Anlehnung an Eggebrecht, bestehe nicht in der Abweichung sondern in der Konkretion von Normen ${ }^{14}$ : Je gelungener ein tonales Werk, desto verdichteter sein tonales System. Die Kultur der Tonalität ermöglicht erst das Meisterwerk, so wie andersherum das Meisterwerk die Kultur der Tonalität beeinflusst.

Die Konsequenzen von Polths Einsichten wären für mich folgende:

1. Ein wichtiges Ziel des Musiktheorieunterrichts ist es, tonale Kompetenz zu erwerben. Wenn die Kultur der Tonalität auf einem bestimmten Usus beruht, so muss ein Kanon tonaler Standards vermittelt werden.

2. Wenn die Kultur der Tonalität in den großen Werken der auctores aprobati prototypisch zu finden ist, folgt daraus die Frage nach dem Kanon der Meisterwerke und der auctores aprobati. Diesen zu finden kann nur gelingen, wenn man nach der tatsächlichen historischen Wirksamkeit fragt und einige Scheuklappen ablegt. Mehr dazu später.

3. Analyse heißt nicht nur, das Singuläre zu suchen und die geniale Organizität des Meisterwerks zu adorieren, sondern das Werk so zu dekomponieren, dass das konkrete und individuelle Funktionieren des Systems Tonalität sichtbar wird. Eine solide Produktionsästhetik bringt die Schüler hinsichtlich ihrer eigenen Produktivität weiter als die ewige Beschwörung der Genieästhetik. Die der Genieästhetik innewohnende Vorstellung, das Meisterwerk zeichne sich vor allem durch Regelbruch aus, ist zutiefst theoriefeindlich, weil Theorie so letztlich die Negativfolie bleiben muss, auf der sich das Werk des Genies glanzvoll abhebt. Eine gelungene Produktionsästhetik hingegen wäre darum bemüht, das Gefüge des Meisterwerks und seine implizite Theorie im historischen Kontext und nicht gegen ihn zu verstehen.

11 Polth 2001.

12 Ebd., 13.

13 Ebd., 34.

14 Ebd. 16. 
Eine Theorie, die den tonalen Usus darstellen will, muss des Weiteren die historischen Quellen ernst nehmen und bereit sein, eingefahrene Denkweisen und Begriffe über Bord zuwerfen. Der tonale Usus nämlich manifestiert sich nicht unbedingt in den Lehrwerken der letzten 50 Jahre, ja nicht einmal der letzten 150 Jahre, sondern vorwiegend in einer Lehrtradition, die vor der Erfindung der so genannten ^Harmonielehre liegt. Das Konzept der `Harmonielehre dem Wunsch, das `Ganze` der Musik möglichst einfach und logisch darzustellen. Wenn man bedenkt, dass die letzten tonalen Komponisten in den 30er und 40er Jahren des 19. Jahrhunderts ausgebildet wurden, so muss sich der historische Focus nach hinten verschieben. Für Dürrnberger, den Lehrer Bruckners vor Sechter, ist ıHarmonielehre` eine Art elementare Musiklehre, während `Generalbass` den Usus festhält, den man als Tonsetzer, Organist und Dirigent beherrschen muss. ${ }^{15}$

Das formallogische Denken der immer spekulativ angehauchten Harmonielehren hat womöglich mehr zur Auflösung von Tonalität als zu deren Konsolidierung beigetragen. ${ }^{16}$ Die formallogischen Harmonielehren orientierten sich letztlich am naturwissenschaftlichen Wahrheitsbegriff - daher auch der ständig wiederkehrende Rekurs auf Helmholtz. Eine konsequente Anwendung formallogischen Denkens aber, etwa die des Umkehrungsdenkens, untergrub letztlich die Fundamente tonaler Harmonik (man denke nur an die konsequente Umkehrung des übermäßigen Quintsextakkordes, etwa bei Bruckner). Dass romantische Harmonik von Kurth schließlich als krisenhaft beschrieben wurde ${ }^{17}$, ist nicht zuletzt auf die praxisferne, ja geradezu autistische Entwicklung der romantischen Harmonielehre zurückzuführen.

Tonalität aber funktioniert nicht formallogisch, sondern eher sprachähnlich. Über den tonalen Usus kann man bei Händel ${ }^{18}$, bei Francesco Durante ${ }^{19}$, bei Fenaroli ${ }^{20}$, in Mozarts Attwood-Studien ${ }^{21}$, bei Albrechtsberger ${ }^{22}$ oder bei Förster ${ }^{23}$, um nur einige Autoren zu nennen, mehr lernen als in allen späteren ^Harmonielehren . In diesem Zusammenhang sei auch auf die Bedeutung der neapolitanischen Konservatorien verwiesen, deren Einfluss auf die barocke Oper, die Wiener Klassik und die italienische Oper des 19. Jahrhunderts gar nicht überschätzt werden kann.

Verfolgt man die Geschichte der Komponistenausbildung, so zeigt sich, dass es einige Denkweisen und Techniken gab, die zwar heute zuweilen eine untergeordnete Rolle spielen, mit Hilfe derer man aber dem tonalen Usus näher kommen könnte. Ich greife -

15 Dürrnberger 1841, IV.

16 Aus diesem Grund kritisierte jüngst Hellmut Federhofer (2006), dass Wolfgang Budday den Begriff `Harmonielehre` überhaupt auf die Schriften Mozarts oder Försters anwendet. `Harmonielehre` steht für Federhofer für den Verfall handwerklicher Kompetenz im 19. Jahrhundert.

17 Kurth 1923.

18 Mann 1978.

19 Durante 2003.

20 Fenaroli 1775.

21 Abgedruckt in Budday 2002.

22 Seyfried 1826.

23 Abgedruckt in Budday 2002. 
in gebotener Kürze - nur drei Dinge heraus: den Außenstimmensatz, den Generalbass und die Improvisation.

Der kontrapunktisch konzipierte Außenstimmensatz beerbt die sars perfecta des Renaissance-Zeitalters und bildet das Gerüst für Klangverbindungen bis in die Spätromantik. Mit dem Aufkommen der als Sopran-Bass-Gerüstsatz konzipierten Monodie und deren dominierender Rolle im Barockzeitalter und weit darüber hinaus, wird der Außenstimmensatz zum kontrapunktischen Paradigma, in dem der salte Kontrapunkt aufgehoben ist und erweitert werden kann. Harmonik ist, von dieser Perspektive aus gesehen, keine Aneinanderreihung von Akkordblöcken, sondern die vertikale Sicht auf die Füllung eines kontrapunktischen Außenstimmensatzes. ${ }^{24}$ Wenn der Außenstimmensatz bei Wagner noch ungebrochen funktioniert und bei Debussy nicht mehr ${ }^{25}$, so werden ein tonales Prinzip und seine Grenzen deutlich sichtbar.

Generalbass ist weit mehr als mechanisches Akkordgreifen. Generalbass ist einerseits eine aufführungspraktische Angelegenheit und ermöglicht andererseits einen dezidiert theoretischen Zugriff. Die Oktavregel ist dabei nur die Spitze des Eisberges. Wie man bei Mozart, Förster, Fenaroli und anderen sehen kann, impliziert er eine komplette Lehre der Klangverbindungen, die den Außenstimmensatz gewissermaßen akkordisch nach innen ausfüllt. Von großem Nutzen ist vor allem die Disziplin des unbezifferten Generalbasses: Wie Akkordverbindungen tonal organisiert werden, kann man hier so gut lernen wie nirgends sonst. Die stilistische Reichweite ist weiter, als immer wieder behauptet wird: So gut wie alle führenden Harmonielehren des 19. Jahrhunderts enthalten Generalbassübungen, einige erfolgreiche Lehrer, wie etwa Josef Gabriel Rheinberger vertrauten ganz auf den Generalbass. Es ist geradezu verblüffend, wie in Rheinbergers Übungen (überliefert sind bezifferte und unbezifferte Bässe) eine spätromanische Harmonik bei ganz übersichtlicher Bezifferung zustande kommt.

Fast alle tonalen Komponisten bis Bruckner waren hervorragende Improvisatoren. Wie man an der neapolitanischen Schule, aber auch bei Franzosen wie etwa Grétry ${ }^{26}$ sehen kann, spielte Improvisation eine entscheidende Rolle in der Ausbildung. Der Begriff ^Improvisation`selbst mag irreführend sein, weil man sich darunter gerne genialischanarchische Ergüsse vorstellt. Sinnvoller ist es vielleicht, von einer sschriftlosen Musizierpraxis« zu sprechen, deren Regelhaftigkeit in einer engen Beziehung zur Komposition steht. ${ }^{27}$ Was tonaler Usus ist, ist hier im wahrsten Sinn ımit Händen zu greifen`. In allen Quellen zur Improvisation, von Guilielmus Monachus bis Grétry, wird deutlich, dass Re-

24 Ich habe versucht, diese Denkweise anhand einer Analyse des ersten Concerto grosso op. 6 von Händel analytisch fruchtbar zu machen (Menke 2007).

25 Diese Anregung verdanke ich Bernnat 2001, 41.

26 Grètry 1801.

27 Zwei Symposien zur Improvisation im Barock sowie in Mittelalter und Renaissance, die 2007 und 2008 an der Schola Cantorum Basiliensis stattgefunden haben, haben eindrücklich gezeigt, dass im gesamten behandelten Zeitraum (und darüber hinaus) schriftlose Musizierpraxis eine bedeutende Rolle spielte; eine Erkenntnis, welche insofern auch ein ganz anderes Verständnis von komponierter Musik nach sich zieht, als die Grenzen zwischen Improvisation und Komposition weitaus durchlässiger sind, als es der genieästhetische Werkbegriff zulässt. 
gelhaftigkeit in Form von Modellhaftigkeit in Verbund mit der Fähigkeit zur Diminution von Gerüstsätzen den wichtigsten Zugang zur Improvisation darstellt.

Die Konsequenz aus diesen Überlegungen zu einem Kanon der Kompetenzen wäre eine neue Ausrichtung bzw. eine neue Konzeption bereits bestehender musiktheoretischer Disziplinen:

- Kontrapunkt bildet als Außenstimmensatz die Grundlage auch für sharmonische` Sätze.

- Generalbass ermöglicht einen sehr direkten Zugriff auf tatsächlich notierte und erklingende Töne (ohne das Grübeln über Grundtöne oder verborgene Fundamente) auch im 19. Jahrhundert.

- Modellorientierte Improvisation macht die regelhafte Verfasstheit von Musik unmittelbar zugänglich. Sie fördert das Bewusstsein, dass Werke nicht nur den singulären Köpfen unsterblicher Meister zu verdanken sind, sondern auch und vor allem einer diese umgebenden lebendigen musikalischen Kultur. Diese Kultur (und nicht nur Meisterwerke) zu tradieren, ist für mich ein wesentliches Movens des Unterrichtens.

Mit welchen Stilen und Gattungen man sich schreibender- und musizierenderweise beschäftigt, kann ganz unkanonisch angegangen werden, wenngleich die Frage nicht ausgespart werden sollte, welche Repräsentanz die gewählte Gattung für die jeweilige Epoche hat. So kann durchaus daran gezweifelt werden, ob die heiligen Kühe Bach-Choral und Bach-Fuge wirklich repräsentativ für das 18. Jahrhundert sind. Die Triosonate (deren Corellischem Muster beinahe alle Komponisten huldigten), die Suite (Ausdruck der höfischen Kultur und des französischen Einflusses) sowie die Arie (Affekte und Rhetorik, italienischer Einfluss) scheinen mir eine weitaus wichtigere Rolle zu besitzen und eignen sich für die Satzlehre genauso gut.

IV.

Eine Modifizierung des Kanons der musiktheoretischen Kompetenzen wirkt sich auch auf den Kanon der Werke aus. Entscheidend hierbei sind das Beiseitelegen von Scheuklappen und die ausbalancierte Synthese von Vielfalt und Repräsentanz. Hierzu möchte ich folgende Punkte zu bedenken geben:

1. Schlüsselfunktion des 17. Jahrhunderts. Harmonische Tonalität, viele Techniken und Gattungen (Mododie, Generalbass, Affektenlehre, Rhetorik etc.) etablierten sich im 17. Jahrhundert. Wie man am Konzertleben, auf dem Tonträgermarkt und in den Medien sehen kann, hat sich das Repertoire hier enorm ausgedehnt. Dem sollte ein Kanon Rechnung tragen.

2. NationaleScheuklappen. Dieoftbeschworene LinieBach-Beethoven-Brahms-WagnerSchönberg ist eine germanozentrische und zutiefst ideologische Konstruktion. Neben Wien, Leipzig und Dresden waren auch Paris, Rom, Neapel, Madrid, London, Budapest und St. Petersburg, um nur einige wichtige Beispiele zu nennen, ebenbürtige und einflussreiche Musikstädte. Das sollte man auch im Musiktheorie-Unterricht spüren. 
3. Vernachlässigung erfolgreicher Komponisten. Schönbergs Diktum, Erfolg besage gar nichts, außer, dass man es sich zu leicht gemacht habe ${ }^{28}$, wurde oft dermaßen verinnerlicht, dass man erfolgreiche Komponisten von vornherein argwöhnisch betrachtete und lieber vermeintliche Außenseiter und Rebellen in den Kanon aufnahm. Erfolgreiche Komponisten wie Carissimi, Lully, Corelli, Alessandro Scarlatti, Händel, Paisiello, Rossini, Cherubini oder auch Meyerbeer und Liszt, um nur einige Namen herauszugreifen, haben den tonalen Usus erheblich geprägt und ihre Kollegen angeregt.

4. Bedeutung der Oper. Seit Monteverdi ist die Oper eine, ja zeitweise die zentrale Gattung gewesen und hat sich entsprechend auf andere Gattungen ausgewirkt. In Hinblick auf die Analyse besteht hier einiger Nachholbedarf. Als Musiktheoretiker neigt man gerne dazu, kurze und `komplexe` Stücke zu bevorzugen, scheut hingegen davor zurück, dezidiert affektbetonte Musik zu behandeln. Dies sind wir aber unseren Schülern und der musikalischen Realität schuldig.

Abschließend sei noch einmal festgehalten: In der Ausbildung kommt man um eine freiwillige oder unfreiwillige Kanonbildung nicht herum. Die Entwicklungen der letzten Jahre haben gezeigt, dass Kenntnisse der historischen Quellen einen ganz anderen Zugriff auf tonales kompositorisches Denken erlauben. Eine der Genieästhetik entspringende Dichotomie von formallogischer Harmonielehre einerseits und individuellen Meisterwerken andererseits verhindert eine gelungene Teilhabe an der Kultur der Tonalität. Darüber, dass Werke mit später kodifizierten und/oder für die Urheber nicht relevanten Normen brechen, muss man sich, nebenbei gesagt, auch nicht wundern. Eine gelungene Teilhabe an der Kultur der Tonalität könnte ermöglicht werden durch einen der Produktionsästhetik verpflichteten Kanon von Kompetenzen, mit deren Hilfe ein operativer Zugriff auf den tonalen Usus möglich wird. Ein produktionsästhetisch motivierter Kanon der Werke hätte sich ideologischer Voreingenommenheiten zu enthalten und als Auswahlkriterium neben der Qualität die Repräsentanz und historische Bedeutung eines Werkes einzubeziehen.

Kanonbildung hat nichts zu tun mit Restriktion, sondern mit Tradition im wörtlichen Sinne: Die Frage nach dem Kanon ist die Frage danach, was wir sweitergeben und dies sollte mehr sein als ewige Meisterwerke und musiktheoretische Systeme. Wenn es uns als Musiktheoretikern gelingen würde, ein Stück der lebendigen Kultur zu tradieren, aus der Meisterwerke hervorgegangen sind und hervorgehen, wäre - nicht nur für unser Fach - viel gewonnen. 


\section{Literatur}

Budday, Wolfgang (2002), Harmonielehre Wiener Klassik, Stuttgart: Berthold \& Schwerdtner.

Bernnat, Andreas (2001), »Tonalität bei Debussy«, Musik \& Ästhetik 18, 37-52.

Dürrnberger, Johann August (1841), Elementar-Lehrbuch der Harmonie- und Generalbaß-Lehre, Linz: K. k. Normal-Hauptschule.

Durante, Francesco (2003), Bassi e Fughe, Padua: Armelin Musica.

Federhofer, Hellmut (2006), »Hat Wolfgang Amadeus Mozart eine Harmonielehre verfaßt?«, Musiktheorie 21/4, 291-299.

Fedele Fenaroli (1775), Regole musicali di cimbalo, Neapel, Reprint Bologna: Forni 1975.

_ (1863), Partimenti, ossia basso numerato, Florenz, Reprint Bologna: Forni 1967.

Gemoll, Wilhelm (1988), Griechisch-Deutsches Schul- und Handwörterbuch, München/ Wien: Teubner.

Grétry, André-Ernest-Modeste (1801), Méthode simple pour apprendre à préluder an peu de temps avec toutes les ressources de l'harmonie, Paris: L'Impremerie de la République.

Fuhrmann, Manfred (2002): Bildung. Europas kulturelle Identität, Stuttgart: Reclam.

Händel, Georg Friedrich (1978), Aufzeichnungen zur Kompositionslehre (= Hallische Händel-Ausgabe, Supplement, Bd. 1), hg. von Alfred Mann, Kassel u. a.: Bärenreiter.

Kurth, Ernst (1923), Romantische Harmonik und ihre Krise in Wagners sTristanı, 3. Aufl. Berlin, Reprint Hildesheim u. a.: Olms 1998.

Menke, Johannes (2007), „Klang, Prozeß und ıSkelett‘. Zum ersten Satz aus Händels Concerto grosso op. 6,1«, Musik \& Ästhetik 44, 54-68.

Polth, Michael (2001), »Nicht System - nicht Resultat«, Musik \& Ästhetik 18, 12-36.

Reich-Ranicki, Marcel (2002), Der Kanon. Die deutsche Literatur, Frankfurt a. M.: Insel.

Rheinberger, Josef Gabriel (2001), Bassübungen für die Harmonielehre, Vaduz: Verlag des Rheinberger-Archivs.

Schönberg, Arnold (1911), Harmonielehre, Wien: Universal Edition.

Schwanitz, Dietrich (1999), Bildung. Alles was man wissen muß, Frankfurt a.M.: Eichborn.

Seyfried, Ignaz Ritter von (1826), J. G. Albrechtsberger's sämmtliche Schriften über Generalbaß, Harmonie-Lehre, und Tonsetzkunst, Wien: A. Strauss. 